\title{
POS-SUPPORTED AUTOMATIC DIGITAL SURFACE MODEL (DSM) GENERATION
}

\author{
He Hongyan ${ }^{\text {a }}$, Zhou nan ${ }^{\text {a, } * \text {, YueChunyu }}{ }^{\text {a }}$ \\ ${ }^{a}$ Beijing Institute of Space Mechanics and Electricity, China - nan_zhou2006@126.com
}

KEY WORDS: POS, DSM, Aerial Image, Dense Matching, Relaxation Optimization

\begin{abstract}
:
A novel method of POS supported dense matching is introduced for automatically generating DSM. The image pre-processing includes adaptive smooth filter and Wallis filter. The method based on the correlation coefficient with geometric constraint, POS supported geometry corrective for matching window, and global relaxation optimization is introduced in the process of matching for determining the homogeneous points. The Multi-image space intersection method with outlier elimination function is used to realize the matching result to integrate into the object space and generate coarse DSM. The matching results of upper pyramid generating a coarse DSM, which is used to constrain and guide the subsequent pyramid image matching, until the original image. Finally, the feasibility of the method proposed in this paper is verified by the experiments, which tests on different scales of aerial images.
\end{abstract}

\section{INTRODUCTION}

Dense matching as the key technology of automatically generating DSM, has always been a research hotspot in the digital photogrammetry and computer vision field. In recent years, although the image matching technology has been extensive researching and further developing, but a universal and effective method to solve all problems that did not appear, which can generate DSM high accurately and automatically. With the aerial digital sensors widely used, they can get large overlap aerial image, so as to providing large redundant geometry and radiation information for image matching, how to take full advantage of the redundant information to obtain point cloud accurately, reliably and automatically, is a focus in the study of this paper. In addition, the POS system is introduced, which can direct determinate the image orientation parameters. How to make use of the POS to support image matching, is worth of discussion.

Gabet etc proposed a method for multi-image matching by synthesizing image and object space information, this kind of method is easy to achieve, but large amount of calculation (Gabet, 1997). Okutomi 、Zhang etc proposed object spacebased image matching to use multi-image redundant information, the method require precise orientation elements (Okutomi, 1997; Zhang, 2004).

Hence, this paper presents a POS-supported multi-image matching method for generating DSM automatically. The basic idea is that matching in the image space, and then through a multi-image space intersection integrated into the object space. For $\mathrm{N}$ images, select an image as the reference image and other images are searching image. The initial disparity between the reference image and each searching image are calculated in the top level of pyramid. The method based on the correlation coefficient with geometric constraints, POS supported geometry corrective for matching window and global relaxation optimization is introduced in the process of matching. Finally, the matching results in all stereo pairs are integrated into the object space basing on multi-image space intersection with iteration method with variable weight eliminating gross errors for DSM. The matching result of upper pyramid image is used to constrain and guide the subsequent pyramid image matching, until the original image and the coarse-to-fine matching strategy can obtain accurate and reliable elevation information.

\section{THE MATCHING APPROACH}

\subsection{Image Pre-processing}

The performance of the image matching depends on the quality of the images. The accuracy and reliability of the image matching result is affected by the image contrast, the image noise and the radiation distortion. In order to reduce the effects of the radiometric problems, a pre-processing is used. The method consists of two steps: the first step, an adaptive smoothing filter is applied to reduce the noise level and preserve even fine detail such as corners (Philippe, 1991). Secondly, the Wallis filter is applied to enhance and sharpen the already existing texture patterns (Zhang, 1999).

\subsection{POS Supported Geometry Corrective for Matching Window}

Due to the perspective and the terrain difference, causing the geometric distortion of the image and bringing some difficulties in image matching. The method of POS supported geometry corrective for matching window is developed to eliminate geometric distortion before image matching. The method requires the POS data and approximate elevation information. Steps as follows:

1. As the POS data and the rough DSM are known, the rough DSM can be assumed as a constant horizontal plane or the upper pyramid image matching results.

2. Around feature points open up a w* w window on the reference image, base on the collinearity equation projected the window onto the object space by the POS data and the rough DSM. Then the object space surface back-project to searching images. So as to eliminate the geometric distortion the image causing by the perspective, 
the terrain and other factors. The matching window of the searching images becomes irregular quadrilateral after that. 3. Using the affine transformation to describe the geometric distortion, and the transform coefficients are solved by the four corners of the quadrilateral.

4. Resampling is applied to the search image, which can eliminate the effect of the geometric distortion for image matching.

\subsection{Correlation Coefficient with Geometric Constraint}

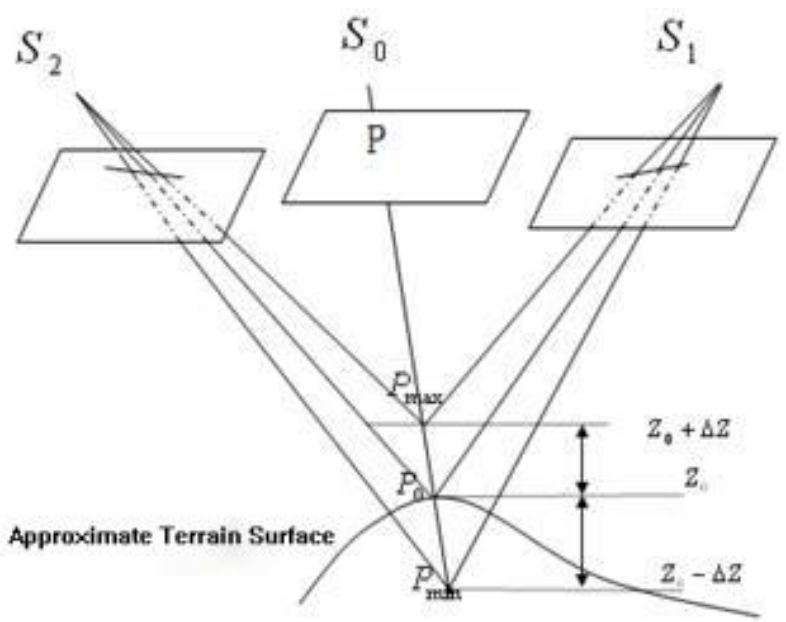

Figure 1 Correlation Coefficient with Geometric Constraint

It is assumed that the image orientation parameters are known, For a given point $P$ in the reference image and the projection center $S_{0}$, we can determine the image ray $S_{0} P$, By intersecting the image ray $S_{0} P$ with the plane defined by a given approximated height $Z_{0}$, we obtain $P_{0}\left(X_{0}, Y_{0}, Z_{0}\right)$ in object space. The approximate elevation is known from the approximate DSM of the upper pyramid image matching result or a constant horizontal plane. Due to the approximate height $Z_{0}$ may not accurate and assumed the certain error $\Delta Z$, the correct elevation position of the point $P$ should be located between $Z_{0}-\Delta Z$ and $Z_{0}+\Delta Z$. If we back-project the points between $P_{\min }$ and $P_{\max }$ onto the search images, the corresponding segments of the epipolar lines (or to be more precise the epipolar curves, but in the first approximation they can be treated as straight lines) for the point $P_{0}$ can be easily defined (Zhang, 2005).

Taking into account the orientation parameters error, the search range is usually from 5 to 8 pixels around the epipolar lines. We select the normalized correlation coefficient as the similarity measurement (threshold=0.5) to determined the match candidates.

\subsection{Global Relaxation Optimization}

In this paper, the probability relaxation algorithm is introduced in the process of matching for determining the optimization homogeneous points. The probability relaxation method considerate context information of the neighborhood and impose a piecewise smoothness surface constraint, so that the correct match gains the highest supports (for details, refer to Chou, 1998; Zheng, 2004).

\subsection{Multi-image Space Intersection and Iteration Method with Variable Weights}

The feature points on the search image may match successfully in some stereo pairs, but fails in others, or exist mismatching. Hence, the matching results in all stereo pairs are integrated into the object space by the multi-image space intersection, which based on the least square method to calculate the ground coordinate. Assume one feature point on the $\mathrm{n}$ search images, and orientation parameters are known, according to the collinearity equation, we can define the error equation as:

$$
\begin{aligned}
& v_{x}=-a_{11} \Delta X-a_{12} \Delta Y-a_{13} \Delta Z-\left(x-x^{0}\right) \\
& v_{y}=-a_{21} \Delta X-a_{22} \Delta Y-a_{23} \Delta Z-\left(y-y^{0}\right)
\end{aligned}
$$

The meaning of symbols refer to $\mathrm{Li}, 1992$.

In the adjustment process, the iteration method with variable weights method is introduced for eliminated mismatching points.

\section{EXPERIMENTAL RESULTS TRANSMITTAL}

The algorithm has been tested on different scale aerial images, and compares the performance of POS and precise orientation parameters supported dense matching. All the images have 7680 x 13824 pixels $(G S D=0.048 \mathrm{~m}, 0.12 \mathrm{~m}$ and $0.288 \mathrm{~m})$, the scales consist of 1:4000, 1:10000 and 1:24000. The test areas have an elevation range of more than $1600 \mathrm{~m}$ and the land cover is extremely variable which contains plains and hills, also has a large number of repeat and lack texture region. The accuracy of

\begin{tabular}{|c|c|c|c|c|c|c|c|c|c|}
\hline \multirow[b]{2}{*}{ Test } & \multirow[b]{2}{*}{ Scale } & \multirow{2}{*}{$\begin{array}{c}\text { Check } \\
\text { point }\end{array}$} & \multirow[b]{2}{*}{ error } & \multicolumn{3}{|c|}{ Accuracy } & \multicolumn{3}{|c|}{ POS } \\
\hline & & & & $X$ & $\mathrm{Y}$ & $\begin{array}{c}\text { Plan } \\
\text { e }\end{array}$ & $X$ & $\mathrm{Y}$ & $\begin{array}{c}\text { Plan } \\
\text { e }\end{array}$ \\
\hline 1 & $1: 4000$ & 215 & RMS & $\begin{array}{l}0 . \\
5\end{array}$ & $\begin{array}{c}0 . \\
8\end{array}$ & 0.9 & $\begin{array}{l}6 . \\
8\end{array}$ & $\begin{array}{l}5 . \\
2\end{array}$ & 8.6 \\
\hline 2 & $\begin{array}{c}1: 1000 \\
0\end{array}$ & 214 & RMS & $\begin{array}{l}0 . \\
6\end{array}$ & $\begin{array}{l}0 . \\
6\end{array}$ & 0.8 & $\begin{array}{l}6 . \\
0\end{array}$ & $\begin{array}{l}7 . \\
4\end{array}$ & 9.6 \\
\hline 3 & $\begin{array}{c}1: 2400 \\
0\end{array}$ & 214 & RMS & 0. & $\begin{array}{l}0 . \\
3\end{array}$ & 0.5 & $\begin{array}{l}3 . \\
8\end{array}$ & $\begin{array}{l}2 . \\
3\end{array}$ & 4.4 \\
\hline
\end{tabular}
the orientation parameters are shown in Table 1.

Table 1. Error of the orientation parameters（in pixel）

\subsection{Different Orientation Parameters Supported Dense Matching Evaluation}

In order to evaluate the dense matching method proposed in this paper, we show the dense matching results based on based on the POS data and accuracy orientation parameters (see Figure 2 and Figure 3).

Table 2 gives the dense matching performance evaluation from the aspect of matching success rate and matching points. 


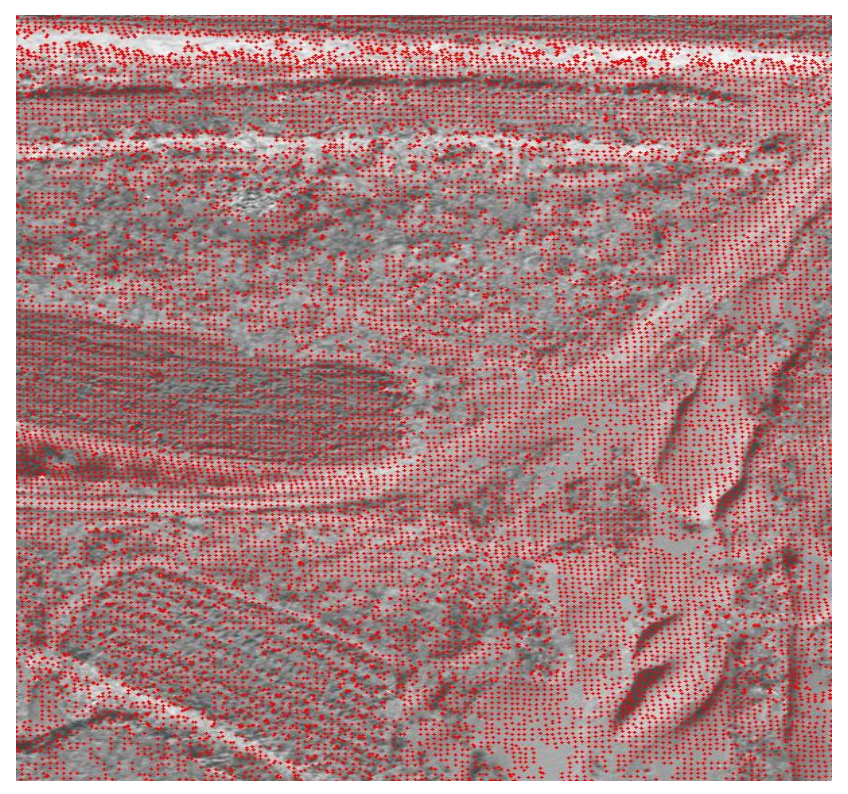

Figure 2. POS-supported dense matching

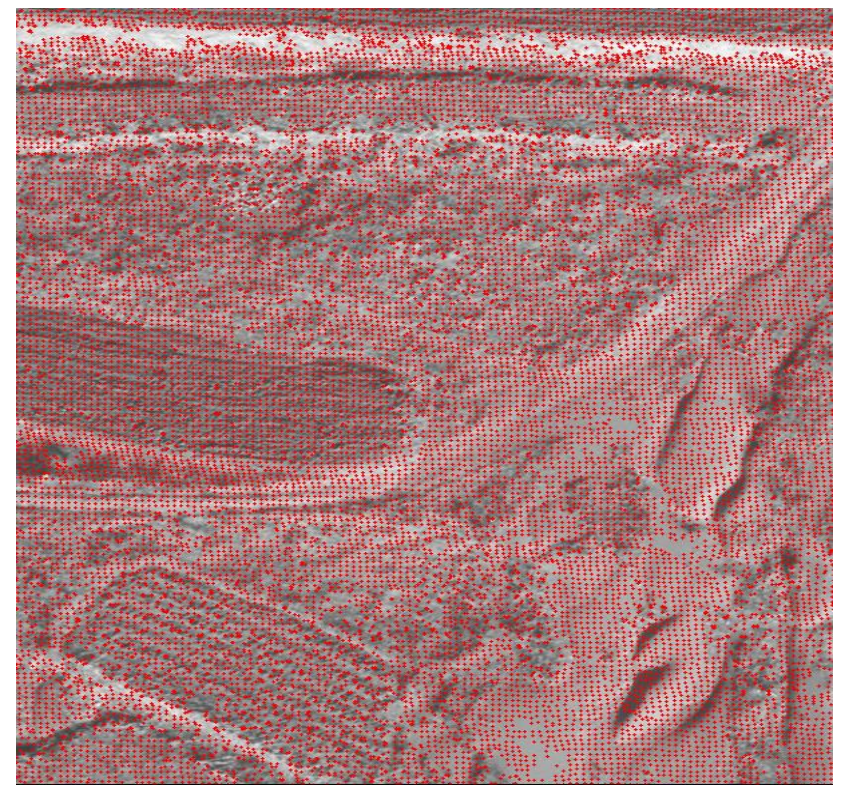

Figure 3. Accuracy-supported dense matching

\begin{tabular}{cccccc}
\hline \multirow{2}{*}{ Test } & \multicolumn{2}{c}{ Number } & & \multicolumn{2}{c}{ Successful Rate } \\
\cline { 2 - 3 } \cline { 5 - 6 } & Accuracy & POS & & Accuracy & POS \\
\hline 1 & 613711 & 453091 & & $97.2 \%$ & $71.4 \%$ \\
2 & 947058 & 847009 & & $95.5 \%$ & $85.4 \%$ \\
3 & 832415 & 774384 & & $87.7 \%$ & $81.6 \%$ \\
\hline
\end{tabular}

Table 2. Dense matching results comparison

The experiment results show that, the matching successful rate of POS is significantly lower than the accuracy orientation parameters. The reason is that the error of POS reduced the accuracy and reliability of the matching results in the image space, and when the matching results are integrated into the object space, the homogeneous points with low accuracy and reliability are eliminated as gross error by multi-image space intersection method.

\subsection{DSM Generation Comparison Test}

In order to verify the feasibility of the method proposed in this paper and compare DSM generation based on the POS data and the accuracy orientation parameters, we use the dense matching algorithm proposed above to obtain the points cloud, and build triangulated irregular network to generate DSM. Figure 2 and figure 3 show the performance of DSM generation based on POS and accuracy orientation parameters.

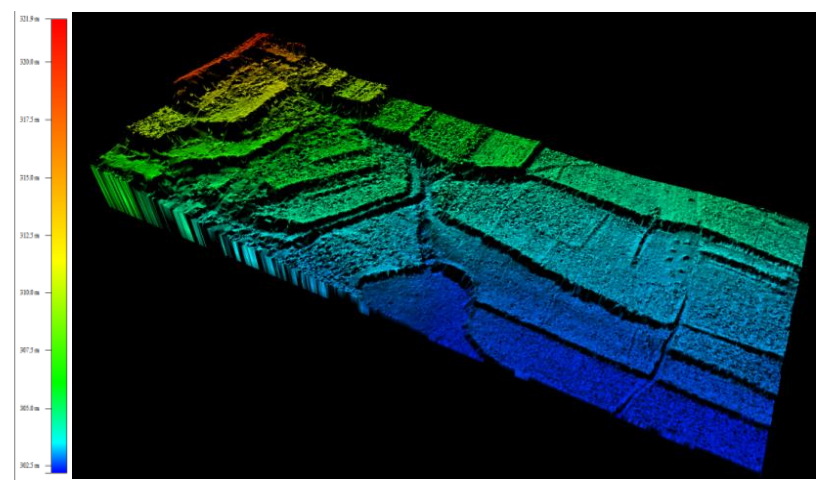

Figure 4 POS-supported generate DSM

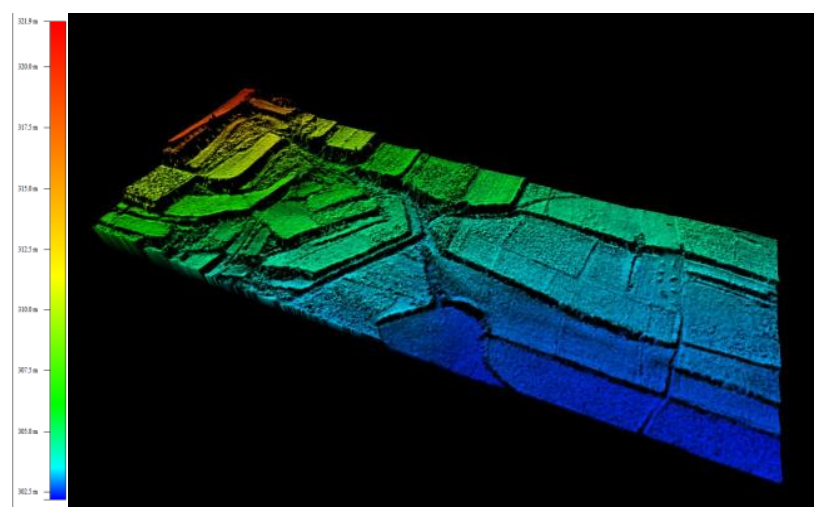

图 2 Accuracy-supported generate DSM

The resulting DSM based on POS and accuracy orientation parameters reproduced quite well not only the terrain relief, but also the small geomorphological features. The DSMs show many topographic details and features like valleys and detailed patterns. For matching difficult region like texture lack area, repeat texture area and shadows can obtain better matching results. The performance of the resulting DSM of accuracy orientation is bad than the resulting DSM of POS

In order to quantitatively evaluate the accuracy of the generated DSM, a number of ground control points (GCPs) were used as the reference data. TIN is interpolated as regular grid DSM by using proper interpolation method. According to GCPs plane coordinate and regular grid DSM, we can interpolate the elevation of each GCP, and compute the elevation RMS by point-by-point comparison. Table 3 gives the DSM accuracy evaluation results.

\begin{tabular}{cccccc}
\hline \multirow{2}{*}{ Test } & \multirow{2}{*}{ Scale } & GCP & RMS & \multicolumn{2}{c}{ DSM (m) } \\
\cline { 5 - 6 } & & & $\begin{array}{c}\text { Accura } \\
\text { cy }\end{array}$ & POS \\
\hline 1 & $1: 4000$ & 25 & Min & -0.001 & 0.628 \\
\hline
\end{tabular}




\begin{tabular}{cccccc}
\hline & & & Max & 0.091 & 0.472 \\
& & & Mean & 0.052 & 0.526 \\
\hline \multirow{3}{*}{2} & \multirow{3}{*}{$1: 10000$} & \multirow{3}{*}{34} & Min & -0.001 & 0.940 \\
& & & Max & -0.304 & -2.540 \\
& & & Mean & 0.120 & 1.170 \\
\hline \multirow{3}{*}{3} & \multirow{3}{*}{$1: 24000$} & \multirow{3}{*}{86} & Min & 0.013 & 0.001 \\
& & & Max & 1.108 & 7.326 \\
& & & Mean & 0.262 & 1.528 \\
\hline
\end{tabular}

Table 3. The accuracy of the DSM comparison

The results can be summarized as follows:

1. The accuracy of the resulting DSM is corresponding to the accuracy of the orientation parameters. This can be expected that the accuracy of the DSM lies on the accuracy of the orientation parameters for this method proposed in the paper.

2. For the resulting DSM of the accuracy orientation parameters, a high accuracy of $0.052 \sim 0.262 \mathrm{~m}$ or even subpixel level can be achieved in flat terrain areas, hills areas and even mountain areas, but In urban and forest areas, the accuracy becomes worse, which is due to the fact that the DSM determined in dense matching may produce many mismatching.

3. For the resulting DSM of the POS, a accuracy of $0.526 \sim 1.528 \mathrm{~m}$ that slightly below than the precision of the POS, which is due to the system error of the POS have a certain influence on the accuracy and reliability of the dense matching.

\section{CONCLUSIONS}

In this paper, we have proposed an universal POS-supported dense matching method for automatic DSM generation from aerial images. It can provide dense, precise and reliable point cloud. The method uses a coarse-to-fine hierarchical strategy with an effective several image matching algorithms to determine the homogeneous points, which consists of the correlation coefficient with geometric constraint, POS supported geometry corrective of matching window and relaxation optimization. The matching results are integrated into the object space intersection, which based on multi-image space intersection with iteration method with variable weights eliminating mismatching points.

We have presented the results of the processing of different scales aerial images over several testfields. The resulting DSMs show that our dense matching method can reproduce not only the general features of the terrain relief, but also detailed features. The accuracy of the resulting DSMs lie on the accuracy of the orientation parameters.

\section{REFERENCES}

Zhang Zuxun, Zhang Jianqing, 2002. Principles of Digital Photogrammetry. Wuhan University Press, pp. 334-340.

Gabet L., Giraudon G., Renouard L, 1997. Automatic Generation of High Resolution Urban Zone Digital Elevation Models. International Journal of Photogrammetry and Remote Sensing, 52 (1), pp. 33-47.

Okutomi M, Kanade T, 1993. A Multiple-baseline Stereo. IEEE Transaction on Pattern Analysis and Machine Intelligence, 15 (4), pp. 353-363.
Zhang, L., Gruen, A., 2004. Automatic DSM generation from linear array imagery data. International Archives of the Photogrammetry, Remote Sensing and Spatial Information Sciences 35 (Part B3), pp. 128 - 133.

Philippe S., Jer S., 1991. Adaptive Smoothing: A General Tool for Early Vision. IEEE Transactions on Pattern Analysis and Machine Intelligence, 13(6), pp. 514-528.

Zhang Li, Zhang Zuxun, Zhang Jianqing, 1999. The Image Matching Based on Wallis Filtering. Wuhan Technical University of Surveying and Mapping Academic Journal, (24)1, pp. 24-27.

Zhang L., 2005. Automatic Digital Surface Model (DSM) Generation from Linear Array Images. ETH, pp. 53-69.

Chou Tong, 1998. Wavelet-based Relaxation Image Matching. Wuhan Technical University of Surveying and Mapping Academic Journal, 23(3), pp. 145-148.

Zheng Shunyi, Zhang Zuxun, Zhang Jianqing, 2004. Image Relaxation Matching Based on Feature Points for DSM Generation. Geo-spatial Information Science, 7(4), pp. 243-248.

Li Deren, Yuan Xiuxiao, 2002 Error Processing and Reliability Theory. Wuhan University Press, pp. 160-180

Li Deren, Zheng Zhaobao, 1992. Principles of Analytical Photogrammetry. Beijing: Surveying and Mapping Press, pp. 321-350. 